\title{
Casimir energy and realistic model of dilute dielectric ball
}

\author{
Valery N. Marachevsky* \\ Department of Theoretical Physics \\ St.Petersburg State University \\ 198904 St.Petersburg, Russia
}

\begin{abstract}
The Casimir energy of a dilute homogeneous nonmagnetic dielectric ball at zero temperature is derived analytically for the first time for an arbitrary physically possible frequency dispersion of dielectric permittivity $\varepsilon(i \omega)$. A microscopic model of dielectrics is considered, divergences are absent in calculations because an average interatomic distance $\lambda$ is a physical cut-off in the theory. This fact has been overlooked before, which led to divergences in various macroscopic approaches to the Casimir energy of connected dielectrics.
\end{abstract}

PACS numbers: 12.20.-m, 12.38.Bx, 34.20.Gj, 31.15.-p

keywords: dipole interaction, Casimir energy, dilute dielectrics

*E-mail: root@VM1485.spb.edu 


\section{Introduction}

In a recent paper [1] we proposed a technique for the calculation of Casimir energies of connected dilute dielectric bodies based on a microscopic structure of dielectrics. An interatomic distance (denoted here by $\lambda$ ) is a physical cutoff within this approach, the Casimir energy is considered in the second order $(\varepsilon(i \omega)-1)^{2}$ - the lowest order for the energy of interaction of atoms constituting the ball. The equivalence of a Casimir energy and a dipoledipole interaction for homogeneous dielectrics of an arbitrary form in the

order $(\varepsilon(i \omega)-1)^{2}$ was proved in [1] on the basis of Lifshitz theory [2] [3], so the energy of a dielectric due to a dipole-dipole pairwise interaction of atoms constituting the ball coincides in this order with the Casimir energy (see a line of reasoning in section 2 of the present paper).

Macroscopic and microscopic approaches to the Casimir energy with emphasis on the case of dilute connected dielectrics are discussed in section 2 . In section 3 we use the technique suggested in [1] to derive for the first time the formula for the Casimir energy of a homogeneous dilute dielectric ball in the order $(\varepsilon(i \omega)-1)^{2}$, valid at zero temperature for an arbitrary physically possible frequency dispersion of the ball dielectric permittivity $\varepsilon(i \omega)$. It is proved that Casimir surface force on a dilute dielectric ball is attractive for any $\varepsilon(i \omega)$. In the hypothetical non-physical limit $\lambda \rightarrow 0$ the Casimir energy is divergent for every model of $\varepsilon(i \omega)$. This explains the fact that without the interatomic distance $\lambda$ every approach to the Casimir energy of connected dielectrics was ill-defined. In fact, in condensed matter physics the interatomic distance often serves as a physical cut-off, which makes expressions finite and well-defined. It is argued in this paper that from this viewpoint the Casimir effect is not different from other areas of condensed matter physics, the interatomic distance $\lambda$ is an important ingredient of the theory of connected dielectrics, it makes the theory finite and all calculations are well-defined.

The Casimir energy of a dilute dielectric ball is still believed to be equal to

$$
E_{1}=\frac{23}{1536 \pi a}(\varepsilon-1)^{2}
$$

which is obviously only a non-dispersive limit of one of the terms in the full expression for the energy (1) (see Eq.(7), where the term (1) is derived from the fourth line of (5)). The full energy expression (5) contains additional terms: volume and surface contributions to the energy, which are the first and second lines of Eq.(5) respectively, as well as the terms on the third line of Eq.(5), which don't depend on the ball radius $a$. Below in the introduction it is our aim to explain why the term (11) is only the large distance contribution 
to the Casimir energy of the ball, discuss the subtle points of some approaches where the term (11) was derived and try to persuade the reader why the correct expression for the Casimir energy of a dilute dielectric ball is given by Eq.(5).

Only when the energy is defined as in (4) and (5), it is equal to the sum of dipole-dipole pairwise interactions between the atoms of the ball. The Casimir-Polder potential

$$
U_{\text {Cas.-Pol. }}=-\frac{23 \alpha_{1}(0) \alpha_{2}(0)}{4 \pi r^{7}},
$$

which is a large distance limit of a dipole-dipole potential (3), valid only for distances $r \gg 1 / \omega_{0}, \omega_{0}$ is a characteristic absorption frequency of atoms, was used instead of a dipole-dipole potential (3) in the calculation [4], where the term (11) was first derived. The calculation [1] correctly extracted the large distance contribution to the energy with a dispersion neglected. However, the calculation [4] was non-dispersive from the outset and thus couldn't yield the contribution to the energy from short distances $\left(r \lesssim 1 / \omega_{0}\right)$ between atoms of the ball. Moreover, the use of a dimensional regularization in [4] concealed the divergences which would appear in the energy expression from the integration over short distances between atoms in the 3-dimensional ball since the minimum distance between atoms of the ball was not introduced in [4].

It is often said that the result (1) is the unique finite Casimir energy. In our opininon, this statement demonstrates the failure to appreciate the physically reasonable definition of the Casimir energy. This should be clear from the line of the following examples. The Casimir energy of two neutral atoms coincides with the energy of a dipole-dipole interaction of these atoms. When an atom is located outside the dielectric of an arbitrary form, then in the dilute approximation the Casimir energy is equal to the sum of dipoledipole interactions between the atom and atoms of the dielectric [1]. For two parallel dielectric slabs it is well known that the Casimir energy in a dilute approximation is equal to the sum of pairwise dipole-dipole interactions of atoms constituting the slabs. For a dilute dielectric ball the sum of pairwise dipole-dipole interactions of atoms constituting the ball is given by Eq.(5), not Eq.(回).

Needless to say that the term (1) itself was really important for development of the theory of the Casimir effect in connected dielectrics since this term has been derived via different techniques [4, 5]. These techniques included both field-theoretic Casimir calculations [5] and the summation of the Casimir-Polder potential in $d$ dimensions [4] discussed above. However, from the field-theoretic approach applied as in [5] one can extract correctly only the large distance contribution to the Casimir energy of the ball, e.g. $E_{1}$. This 
large distance contribution $E_{1}$ was found to be the same when summing up the Casimir-Polder potential (2) between atoms of the ball [4] and when the Casimir energy was derived by field-theoretic calculations [5] - so the equivalence of large distance parts of the Casimir energy of a dilute dielectric ball derived by microscopic and macroscopic approaches was proved. It is important to stress that up to now field-theoretic methods didn't yield satisfactorily short distance contributions to the Casimir energy of connected dielectrics. The reason is simple: these methods were developed for disjoint, not connected dielectrics, and application of these methods to connected dielectrics without any changes inevitably leads to different types of divergences in $e v$ ery field-theoretic calculation of the Casimir energy, these divergences are reminiscents of the ill-defined short distance structure of the theory. In this paper we do not aim to present the field-theoretic macroscopically correct derivation of the ball Casimir energy. We rather wish to present for the first time the correct microscopic derivation of the Casimir energy of a dielectric ball in a dilute approximation - in the order $(\varepsilon(i \omega)-1)^{2}$.

So the object of study is a dispersive dielectric ball of radius $a$ at zero temperature which is dilute, i.e. the permittivity $\varepsilon(i \omega)$ of the ball satisfies the condition $\varepsilon(i \omega)-1 \ll 1$ for all frequencies $\omega$. The Casimir energy is calculated in the second order $(\varepsilon(i \omega)-1)^{2}$. A dipole-dipole potential yields Casimir-Polder and van der Waals potentials in the large and short distance limits respectively, we adopt this terminology. We put $\hbar=c=1$ and use rationalized Gaussian units for the Maxwell field where polarizability $\alpha(i \omega)$ is defined by $\varepsilon(i \omega)-1=4 \pi \rho \alpha(i \omega), \rho$ is a number density of atoms.

\section{Macroscopic and microscopic approaches to Casimir energy}

The study of Casimir energy for spherical dielectrics began with the work by Boyer [6] where he derived Casimir energy of perfectly conducting spherical shell. Numerically it is equal to $\simeq 0.09235 / 2 a[7]$. The study of a dielectric ball and its Casimir energy for an arbitrary permittivity began with the work by Milton [8] and continues up to now. Different mathematical methods were used in the Casimir effect calculations [9]. We are not planning to give a description of these methods here, we rather wish to emphasize the main problem in a macroscopic formulation of the theory, discuss the reason for its appearance, outline the solution for dilute dielectrics and the area for further research.

For dispersive dielectrics a macroscopic formulation of the Casimir ef- 
fect was originally proposed by Lifshitz [2] and developed by Dzialoshinskii, Pitaevskii and others [3]. In the macroscopic formulation the system is usually described by the position and frequency dependent dielectric permittivity $\varepsilon(\omega, \mathbf{r})$. For disjoint dielectrics the theory worked perfectly. The well known case of dielectric parallel plates became the classic example [3]. However, serious problems were found when this theory was applied to connected dielectrics. Problems were first found in the case of a homogeneous nonmagnetic dielectric ball described by a dielectric permittivity $\varepsilon(\omega)$ [8]. In calculations performed according to Lifshitz approach and its modifications, cut-off dependent terms were found to survive in all regularization schemes (see the Appendix in [1], for example). Only in the special case of the magnetic dielectric ball satisfying the condition $\varepsilon \mu=1, \mu$ is a magnetic permeability, Brevik and Kolbenstvedt found that cut-off terms don't appear in the theory [10]. The physical reason for appearance of cut-off dependent terms remained unclear for a long period. Hoye and Brevik argued in [11] that the problem has its origin in a continuum description of dielectric media and can be resolved only in a proper microscopic model involving minimum separation between molecules.

From the microscopic viewpoint the Casimir energy of a dielectric media is usually assumed to arise due to many-body interatomic interactions of neutral atoms. In the model considered in the current paper all atoms constituting a dielectric ball are separated by distances greater than $\lambda$. This is why if one calculates the total ball energy due to some realistic potential between atoms of the ball, one always gets the finite energy. The question is which potential between atoms should be used. The following line of reasoning is appropriate for dilute connected dielectrics [四]. For the system consisting of a homogeneous dielectric with the permittivity $\varepsilon(i \omega)$ and a particle (atom) with the polarizability $\alpha(i \omega)$ located outside this dielectric it was rigorously proved in [1] that the Casimir energy of this system (the part of it describing interaction of the atom with a dielectric body in the lowest order $(\varepsilon(i \omega)-1) \alpha(i \omega))$ coincides with the sum of pairwise interactions via a dipole-dipole potential (3) between the atom and the dielectric body. For the model of a dielectric ball considered in this paper one can imagine an atom inside the ball and draw a sphere of radius $\lambda$ around this atom. Outside this sphere a dilute dielectric can be described well by $\varepsilon(i \omega)$. Casimir energy of interaction between an atom and a dielectric located outside the $\lambda$-sphere is equal in the order $(\varepsilon(i \omega)-1) \alpha(i \omega)$ to the sum of dipole-dipole pairwise interactions between the atom and points of the ball outside the $\lambda$-sphere. To find the total Casimir energy of atoms constituting the ball in the order $(\varepsilon(i \omega)-1)^{2}$, one has to perform this trick for every atom of the ball, sum over all atoms and finally multiply this sum by a factor $1 / 2$. This procedure 
is applied to a dilute dielectric ball in the next section. The interatomic distance $\lambda$ is a physical cut-off.

Recently the microscopic approach based on two-point correlation functions and form-factors of different bodies was developed by Barton [12]. The leading terms for short and long distance contributions to the Casimir energy of different bodies were derived by Barton by use of two-point correlation functions in the case of a simple-harmonic oscillator model for dielectric permittivity. The leading terms for arbitrary model of dielectric permittivity in the case of a dilute ball can be obtained from our formula (5); in the special case of a simple-harmonic oscillator model for dielectric permittivity they are in agreement with Barton.

In the next section it is proved that Casimir surface force on a dielectric ball in the order $(\varepsilon(i \omega)-1)^{2}$ is attractive. For a perfectly conducting spherical shell the force is repulsive. Brevik and Kolbenstvedt observed [10 that energy of a perfectly conducting spherical shell can be derived from the energy of a magnetic dielectric ball satisfying $\varepsilon \mu=1$ in the limit $\mu \rightarrow 0$. One may ask a question. If we first put $\mu=1$ and after that perform the limit $\varepsilon \rightarrow \infty$, will we obtain the repulsive force or the attractive contribution will be dominating ? It is impossible to answer this question at the moment, the theory for arbitrary $\varepsilon(i \omega)$ still doesn't exist for connected dielectrics. Microscopic structure of materials should be an important element of such a theory, though the complete theory may probably be developed only at the intersection of macroscopic and microscopic approaches to the ground state energy.

\section{Casimir energy of dilute ball}

In this section we first calculate the Casimir energy of neutral atoms constituting a ball of radius $a$ due to a dipole-dipole pairwise interaction. Then we prove that Casimir surface force is attractive for arbitrary physically possible dielectric permittivity $\varepsilon(i \omega)$ of the ball.

A dipole-dipole interaction of two neutral atoms with atomic polarizabilities $\alpha_{1}(i \omega)$ and $\alpha_{2}(i \omega)$ is described by the potential [13]

$U(r)=-\frac{1}{\pi r^{2}} \int_{0}^{+\infty} \omega^{4} \alpha_{1}(i \omega) \alpha_{2}(i \omega) e^{-2 \omega r}\left[1+\frac{2}{\omega r}+\frac{5}{(\omega r)^{2}}+\frac{6}{(\omega r)^{3}}+\frac{3}{(\omega r)^{4}}\right] d \omega$,

where $r$ is a distance between two atoms. The energy calculation is illustrated by Fig.1. Suppose that an atom (a molecule) with an atomic polarizability $\alpha(i \omega)$ is located at the point $B$. One has to integrate interaction of an atom 

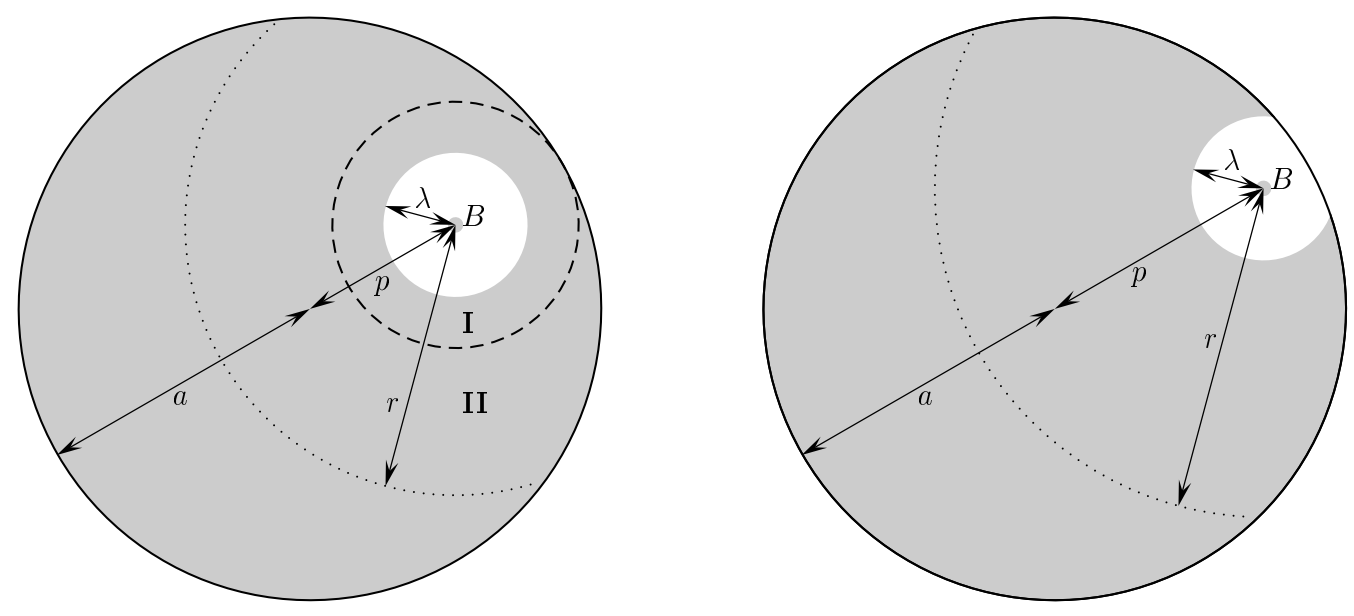

Fig. 1: Energy calculation for the ball. Shaded areas I and II separated by a dashed line on the left picture denote areas of integration in the first and second integrals in (4) respectively. A shaded area on the right picture represents the area of integration in the third integral in (四). A part of the sphere of radius $r$ which is located inside the dielectric ball is denoted by a dotted line. Its area is equal to $2 \pi r^{2}\left(1-\left(p^{2}+r^{2}-a^{2}\right) /(2 p r)\right)$.

at the point $B$ via a potential (3) with the atoms separated by distances greater than interatomic distances $\lambda$ from the point $B$, integrate over all atom locations $B$ inside the ball and multiply by a factor $1 / 2$ to calculate the energy. Assuming homogeneity of the ball (it results in $\alpha_{1}(i \omega)=\alpha_{2}(i \omega)=$ $\alpha(i \omega)$ and the condition that the number density of atoms $\rho$ doesn't depend on the point inside the ball), the Casimir energy is equal to

$$
\begin{aligned}
& E=\frac{\rho^{2}}{2}\left(\int_{0}^{a-\lambda} d p 4 \pi p^{2} \int_{\lambda}^{a-p} d r 4 \pi r^{2} U(r)+\right. \\
& \int_{0}^{a-\lambda} d p 4 \pi p^{2} \int_{a-p}^{a+p} d r 2 \pi r^{2}\left(1-\frac{r}{2 p}-\frac{p^{2}-a^{2}}{2 p r}\right) U(r)+ \\
&\left.\int_{a-\lambda}^{a} d p 4 \pi p^{2} \int_{\lambda}^{a+p} d r 2 \pi r^{2}\left(1-\frac{r}{2 p}-\frac{p^{2}-a^{2}}{2 p r}\right) U(r)\right)
\end{aligned}
$$


Performing calculations, it is straightforward to obtain

$$
\begin{aligned}
E= & -\rho^{2} \frac{\pi}{48} \int_{0}^{+\infty} d \omega \alpha^{2}(i \omega)\left(\frac{a^{3}}{\lambda^{3}} e^{-2 \omega \lambda}\left(128+256 \omega \lambda+128 \omega^{2} \lambda^{2}+64 \omega^{3} \lambda^{3}\right)-\right. \\
& -\frac{a^{2}}{\lambda^{2}}\left(e^{-2 \omega \lambda}\left(144+288 \omega \lambda+120 \omega^{2} \lambda^{2}+48 \omega^{3} \lambda^{3}\right)-96 \omega^{2} \lambda^{2} E_{1}(2 \omega \lambda)\right)+ \\
& \left(e^{-2 \omega \lambda}\left(41+34 \omega \lambda+14 \omega^{2} \lambda^{2}+4 \omega^{3} \lambda^{3}\right)+24 E_{1}(2 \omega \lambda)\right)+ \\
& \left.\left(e^{-4 \omega a}(-21+12 \omega a)-E_{1}(4 \omega a)\left(24+96 \omega^{2} a^{2}\right)\right)\right),
\end{aligned}
$$

where $E_{1}(x)=\int_{1}^{+\infty} e^{-t x} / t d t$.

The formula (5) answers one question which has longly been discussed. The question was: is it possible to make the Casimir energy of connected dielectrics finite (work without divergences) by selecting the proper model of a frequency dependent dielectric permittivity $\varepsilon(i \omega)$ (with a quickly decreasing behaviour when $\omega \rightarrow \infty)$ in a macroscopic approach without other assumptions ? The answer is : there is no such a model. Only when a finite separation between atoms $\lambda$ is taken into account, the energy is finite and physical. When $\lambda \rightarrow 0$, the leading term in (可) ( $V$ is a ball volume) is

$$
-\rho^{2} \frac{2 V}{\lambda^{3}} \int_{0}^{+\infty} d \omega \alpha^{2}(i \omega),
$$

so if we wish to take the hypothetical limit $\lambda \rightarrow 0$, we obtain a divergence for every model of $\alpha(i \omega)$. The leading term (6) can also be obtained directly from the van der Waals limit of (3) for every connected dielectric.

The structure of (5) clearly shows that 4 types of terms contribute to the energy. First and second lines of (5) represent volume and surface contributions to the energy respectively. The third line consists of terms which don't depend on the radius of the ball. The fourth line consists of terms which don't depend on the atomic separation $\lambda$ and depend only on the ball radius $a$.

According to general properties of dielectric permittivity on an imaginary axis, it is possible to write the leading contribution from the fourth line of (5) as (it comes from frequencies $\omega \ll \omega_{0}, \omega_{0}$ is a characteristic absorption frequency of materials, $\omega_{0} a \gg 1$, so it is possible to use the static polarizability $\alpha(0)$ in the leading approximation to the fourth line of (5) )

$$
\begin{array}{r}
-\rho^{2} \alpha^{2}(0) \frac{\pi}{48} \int_{0}^{+\infty} d \omega\left(e^{-4 \omega a}(-21+12 \omega a)-E_{1}(4 \omega a)\left(24+96 \omega^{2} a^{2}\right)\right)= \\
=\rho^{2} \alpha^{2}(0) \frac{23}{96} \frac{\pi}{a}
\end{array}
$$


A review and references to different approaches which had been used to derive (77) are given in the Appendix of [1].

However, the term (17) has a small influence on physics because physics is mainly governed by (6) and next to leading terms in (5). The reason is quite simple - the term (7) is much less in magnitude than the terms in the first, second and third lines of (5). For example, ratio of terms in the first line of (5) to (7) is $\sim\left(\omega_{0} a\right)(a / \lambda)^{3} \gg 1$. Usually all terms but (7) which appeared in different renormalization schemes were simply discarded or considered in macroscopic approaches as divergent contributions which add up to macroscopic quantities of the ball (e.g. volume, surface energies) during the renormalization procedure, as it often happens in field theory. So only the term (7) was usually considered as the Casimir energy term. The coefficient $a_{2}$ in the asymptotic expansion of the heat kernel for a nondispersive dielectric ball in the order $(\varepsilon-1)^{2}$ vanishes [14], this explains the fact that no logarithmic terms appeared in macroscopic calculational schemes for the ball energy, so it was possible to separate the term (7) uniquely from the divergent part of the energy. Theoreticians got used to believe that the Casimir energy should depend only on geometry of the bodies, not on their internal structure, the term (17) depended only on the ball radius $a$, so it seemed to be a proper Casimir energy term. However, Casimir surface force resulting from (17) is repulsive. This consequence seemed unusual but possible. Only the equivalence of the Casimir energy and a dipole-dipole interaction for homogeneous dielectrics in the order $(\varepsilon(i \omega)-1)^{2}$, proved in [1] on the basis of Lifshitz theory, makes obvious the fact that Casimir surface force should be attractive. The potential (3) of a dipole-dipole interaction is attractive for all distances, and one can not obtain repulsive Casimir forces for dilute dielectrics in principle because one starts from the attractive potential. Even from this argument it is clear that the term (17) is not enough to obtain physically meaningful results. Terms depending on the interatomic distance $\lambda$ are greater in magnitude than (7) and must be taken into account in the derivation of Casimir surface force.

After these remarks it is natural to prove that Casimir surface force on a dilute dielectric ball is attractive. It is convenient to define $N \equiv a / \lambda, p \equiv \omega \lambda$. Then the formula (5) can be rewritten in a general form

$$
E=-\frac{\rho^{2}}{\lambda} \int_{0}^{+\infty} d p \alpha^{2}\left(i \frac{p}{\lambda}\right) f(N, p)
$$

The function $f(N, p)>0$ for $N>1, p>0$. The ball expands or collapses homogeneously, so

$$
N=\text { const. }
$$


Conservation of atoms inside the ball imposes the condition

$$
\rho \frac{4 \pi a^{3}}{3}=\text { const } .
$$

It is convenient to use Kramers-Kronig relations in the form

$$
\alpha(i \omega)=\int_{0}^{+\infty} d x \frac{x g(x)}{x^{2}+\omega^{2}},
$$

where the condition $g(x)>0$ always holds [15]. Using (8), (9), (10), (11), Casimir force on a unit surface is equal to

$$
\begin{aligned}
F & =-\frac{1}{4 \pi a^{2}} \frac{\partial E}{\partial a}= \\
& =-\frac{\rho^{2}}{4 \pi a^{3}} \int_{0}^{+\infty} d \omega \int_{0}^{+\infty} d x \frac{x\left(7 x^{2}+3 \omega^{2}\right) g(x)}{\left(x^{2}+\omega^{2}\right)^{2}} \alpha(i \omega) f(N, \omega \lambda)<0,
\end{aligned}
$$

$F<0$ because all functions inside integrals are positive. Casimir surface force is attractive for every model of atomic polarizability consistent with general causal requirements.

It may be worth imagine a model of a dilute dielectric ball which wouldn't exist at all without a dipole-dipole interaction. Without a dipole-dipole interaction atoms would be free at large separations, they would interact only during the collisions due to short range interatomic forces. Energy of a dipole-dipole interaction of such a system is just the energy which holds atoms of the ball together. When the energy due to a dipole-dipole atomic interaction is equal to the repulsive energy due to short range interatomic forces, the system is in equilibrium and stable.

\section{Summary}

The Casimir energy of a dilute homogeneous nonmagnetic dielectric ball at zero temperature is derived for the first time for an arbitrary physically possible frequency dispersion of a dielectric permittivity in the order $(\varepsilon(i \omega)-$ $1)^{2}$ by summing up dipole-dipole interactions between atoms constituting the ball. All calculations are performed without divergences because average interatomic distance $\lambda$ is a physical cut-off. Casimir surface force is proved to be attractive. 


\section{Acknowledgments}

Author acknowledges valuable discussions and correspondence with Gabriel Barton, Michael Bordag, Iver Brevik, Yuri Novozhilov and Dmitri Vassilevich.

\section{References}

[1] V.N.Marachevsky , Casimir energy and dilute dielectric ball , hep-th/0010214, to appear in Physica Scripta.

[2] E.M.Lifshitz, Zh.Eksp.Theor.Fiz.29, 94 (1955).

[3] E.M.Lifshitz and L.P.Pitaevskii, Statistical Physics, Part 2 (Course of Theoretical Physics, vol. IX), (Moscow, Nauka, 1978), Chapter 8.

[4] K.A.Milton and Y.J.Ng, Phys.Rev.E 57, 5504 (1998).

[5] I.Brevik and V.Marachevsky, Phys.Rev.D 60, 085006 (1999).

I.Brevik, V.N.Marachevsky and K.A.Milton, Phys.Rev.Lett.82, 3948 (1999), hep-th/9810062.

G.Barton, J.Phys. A 32 , 525 (1999).

[6] T.H.Boyer, Phys.Rev. 174, 1764 (1968).

[7] K.A.Milton, L.L.DeRaad,Jr. and J.Schwinger, Ann.Phys.(N.Y.) 115, 388 (1978).

[8] K.A.Milton, Ann.Phys.(N.Y) 127, 49 (1980).

[9] Some articles presenting various aspects and techniques in Casimir effect:

Yu.S.Barash and V.L.Ginzburg, Usp.Fiz.Nauk 116, 5 (1975).

K.A.Milton, L.L.DeRaad,Jr. and J.Schwinger, Ann.Phys.(N.Y.) 115, 1 (1978).

M.Bordag, K.Kirsten and D.V.Vassilevich, J.Phys.A 31, 2381 (1998), hep-th/9709084.

V.V.Nesterenko and I.G.Pirozhenko, Phys.Rev.D 60, 125007 (1999), hep-th/9907192.

I.Klich, Phys.Rev.D 61, 025004 (2000), hep-th/9908101. 
J.S.Høye, I.Brevik and J.B.Aarseth, The Casimir problem of Spherical Dielectrics: Quantum Statistical and Field Theoretical Approaches, quant-ph/0008088.

H.Falomir, K.Rébora and M.Loewe, Phys.Rev.D 63, 025015 (2001), hep-th/0008251.

H.Falomir, K.Kirsten and K.Rébora, Divergencies in the Casimir energy for a medium with realistic ultraviolet behaviour, hep-th/0103050.

E.M.Santangelo, Evaluation of Casimir energies through spectral functions, hep-th/0104025.

[10] I.Brevik and H.Kolbenstvedt, Ann.Phys.(N.Y.) 143, 179 (1982); 149, 237 (1983).

[11] J.S.Høye and I.Brevik, J.Stat.Phys. 100, 223 (2000), quant-ph/9903086.

[12] G.Barton, Perturbative Casimir energies of dispersive spheres, cubes, and cylinders, preprint, November 2000.

[13] V.B.Berestetskii, E.M.Lifshitz and L.P.Pitaevskii, Quantum Electrodynamics (Course of Theoretical Physics, vol. IV ), third ed., (Moscow, Nauka, 1989), Chapter 9, eq.(85.17).

[14] M.Bordag, K.Kirsten and D.V.Vassilevich, Phys.Rev.D 59, 085011 (1999), hep-th/9811015.

[15] L.D.Landau and E.M.Lifshitz, Electrodynamics of continuous media (Course of Theoretical Physics, vol. VIII), second ed., (Moscow, Nauka, 1982), Chapter 9, eq.(82.15). 